\title{
Recent patents in endonucleases and genome editing
}

Patent number

Description

Assignee

Inventor

Date

US 10,047,366 Methods and compositions useful for inducibly linearizing circular DNA molecules in vivo in yeast. In one embodiment, the composition comprises an intron comprising an endonuclease recognition site flanked by telomere seed sequences.

US 9,981,020 A method of inactivating a proviral DNA integrated into the genome of a host cell latently infected with a retrovirus by treating the host cell with a composition comprising a CRISPR-associated endonuclease and two or more different guide RNAs (gRNAs), wherein each of the at least two gRNAs is complementary to a different target nucleic acid sequence in a long terminal repeat (LTR) in the proviral DNA, and inactivating the proviral DNA. A composition for use in inactivating a proviral DNA integrated into the genome of a host cell latently infected with a retrovirus including isolated nucleic acid sequences comprising a CRISPR-associated endonuclease and a gRNA, wherein the gRNA is complementary to a target sequence in a human immunodeficiency virus.

US 9,943,612 Polynucleotides having a plurality of thymine nucleotides and an endonu-

US 9,637,739 Isolation or in vitro assembly of the Cas9-crRNA complex of the clease recognition site inserted therein, methods of engineering the polynucleotides having a plurality of thymine nucleotides and an endonuclease recognition site inserted therein, and methods of enhancing transcription and translation, and increasing stability of a polynucleotide.

US 9,944,933 Compositions and methods for modifying genetic material. One embodiment provides aptamers capable of binding to a site-specific DNA binding moiety to facilitate the exchange of homologous genetic information between a donor molecule and the desired target locus (aptamer-guided gene targeting or AGT). One embodiment provides an oligonucleotide containing an aptamer, preferably a DNA aptamer at the 5' end. The oligonucleotide also contains a region of homology, also referred to as donor DNA, to a desired nucleic acid, locus, or gene. The DNA binding moiety can be a nucleic acid, a protein, or a complex of proteins. In a preferred embodiment the DNA binding moiety is a homing endonuclease that cuts DNA to facilitate the modification of the DNA by the donor DNA.

US 9,879,270 Constructs for genome editing or genetic engineering in fungi or protists, methods of using the constructs and media for use in selecting cells. The construct includes a polynucleotide encoding a thymidine kinase operably connected to a promoter, suitably a constitutive promoter; a polynucleotide encoding an endonuclease operably connected to an inducible promoter; and a recognition site for the endonuclease. The constructs may also include selectable markers for use in selecting recombinations.

US 9,840,702 Modified compositions for use in CRISPR systems, and their methods of use. In particular, length-modified and chemically modified forms of crRNA and tracrRNA are described for use as a reconstituted guide RNA for interaction with Cas9 of CRISPR systems. The resultant lengthmodified and chemically modified forms of crRNA and tracrRNA are economical to produce and can be tailored to have unique properties relevant to their biochemical and biological activity in the context of the CRISPR-Cas 9 endonuclease system. Streptococcus thermophilus CRISPR3-Cas system and use for cleavage of DNA bearing a nucleotide sequence complementary to the crRNA and a protospacer-adjacent motif. Methods for site-specific modification of a target DNA molecule using an RNA-guided DNA endonuclease comprising at least one RNA sequence and at least one of an RuvC active site motif and an $\mathrm{HNH}$ active site motif.

US 9,340,800 Compositions, methods, systems and kits for controlling the activity and/ or improving the specificity of RNA-programmable endonucleases, such as Cas9. For example, gRNAs that are engineered to exist in an 'on' or 'off' state, which control the binding and hence cleavage activity of RNAprogrammable endonucleases. Some aspects of this disclosure provide gRNAs that modulate the activity of an RNA-programmable endonuclease
Seattle Children's Hospital (Seattle, WA, USA)

Georgia Tech Research Corp. (Atlanta)
Scharenberg AM,

Jacoby K, Grier AE

$4 / 17 / 2018$

Storici F, Ruff P

Wisconsin

Alumni Research

Foundation

(Madison, WI, USA)

Alexander WG

$1 / 30 / 2018$

Integrated DNA

Technologies

(Coralville, IA

USA)

Vilnius University

(Vilnius, Lithuania)

Siksnys V, Gasiunas G,

Karvelis T

$5 / 2 / 2017$

ngwood MA

Jacobi AM, Rettig GR,

Schubert MS, Behlke MA

based on the presence or absence of an extended DNA (xDNA).
President and

Fellows of Harvard College

(Cambridge, MA, USA)

Source: United States Patent and Trademark Office (http://www.uspto.gov). 